\title{
Mediating effect of waist:height ratio on the association between BMI and frailty: the Korean Frailty and Aging Cohort Study
}

\author{
Minseo $\mathrm{Kim}^{1} \dagger$, Yunhwan Lee ${ }^{2} \dagger$, Eun-Young $\mathrm{Kim}^{1}$ and Yongsoon Park ${ }^{1 *}$ \\ ${ }^{1}$ Department of Food and Nutrition, Hanyang University, Seoul, Republic of Korea \\ ${ }^{2}$ Department of Preventive Medicine and Public Health, Ajou University School of Medicine, Suwon, Republic of Korea \\ (Submitted 22 March 2019 - Final revision received 11 July 2019 - Accepted 6 August 2019 - First published online 27 August 2019)
}

\section{Abstract}

Body weight is a major risk determinant of frailty, but the effect of obesity on frailty is controversial. The present study aimed to confirm the hypothesis that the risk of frailty is positively associated with obesity (BMI $\geq 30 \mathrm{~kg} / \mathrm{m}^{2}$ ), but the association is mediated by the waist:height ratio (WHtR) in older women and men. A total of 2862 community-dwelling older individuals aged 70-84 years were assessed for frailty using the Korean version of Fatigue, Resistance, Ambulation, Illnesses, and Loss of weight index. Obesity (BMI $\geq 30 \mathrm{~kg} / \mathrm{m}^{2}$ ) was associated with a higher risk of frailty compared with BMI $18 \cdot 5-<23 \mathrm{~kg} / \mathrm{m}^{2}$ in all the older individuals (OR 1.88; $95 \% \mathrm{CI} 1 \cdot 11,3 \cdot 17 ; P=0 \cdot 018$ ) and in older women (OR 1.86; $95 \%$ CI 1.01, 3.42; $P=0.047$ ) before adjusting for WHtR but was not associated with BMI after adjusting for WHtR. Additionally, obesity was not significantly associated with the risk of frailty before and after adjusting for WHtR in older men. Mediation analysis revealed that the association between BMI and frailty score was mediated by WHtR. Moreover, the mediating effect of WHtR on frailty score was positive in both women and men, but the frailty score was associated with BMI positively in women and negatively in men. The present study suggests that the risk of frailty is higher in obese women, which is mediated by WHtR, but not in obese men.

\section{Key words: BMI: Older adults: Frailty: Mediation analysis: Waist:height ratio}

Frailty is a state of high vulnerability for adverse health outcomes, including disability, dependency, falls, need for longterm care and mortality ${ }^{(1)}$. Korea is one of the most rapidly ageing countries worldwide ${ }^{(2)}$, and the prevalence of frailty defined by the Korean version of the Fatigue, Resistance, Ambulation, Illnesses, and Loss of weight (K-FRAIL) index has been reported to be $12.4 \%$ in Koreans aged 70 years or older $^{(3)}$.

One of the important factors for frailty is weight loss since the decline in body weight leads to frequently observed underweight in the older adults aged 70 years or older ${ }^{(4,5)}$. Epidemiological studies consistently reported that the prevalence of frailty was higher in the older adults who are underweight (BMI $\left.<20 \mathrm{~kg} / \mathrm{m}^{2}\right)^{(6-10)}$ and lower in those who are overweight (BMI $\left.25-29.9 \mathrm{~kg} / \mathrm{m}^{2}\right)^{(8-10)}$. However, the association between obesity and frailty is controversial. The previous studies showed that obesity defined by BMI $\geq 30 \mathrm{~kg} / \mathrm{m}^{2}$ was positively associated with the prevalence and incidence of frailty in the older adults ${ }^{(8,10-16)}$, but a few studies showed no significant association between obesity and the risk of frailty ${ }^{(7,9,16)}$.
Garcia-Esquinas et al. ${ }^{(16)}$ reported that obesity was positively associated with the risk of frailty in the older adults, but the association was not significant after adjusting for waist circumference (WC), suggesting that the association between BMI and frailty could be mediated by abdominal obesity. A few previous studies reported that abdominal obesity was associated with higher prevalence and incidence of frailty in the older adults ${ }^{(8,9,16,17)}$. Increased WC has been suggested to be associated with a marker of oxidative stress and inflammation, independent of $\mathrm{BMI}^{(18)}$, which may be the major mechanism leading to frailty ${ }^{(19)}$.

Abdominal obesity is commonly defined by WC, and waist: height ratio (WHtR) has been suggested to be a better predictor of whole-body fat percentage and visceral adipose tissue mass as well as increased health risk compared with $\mathrm{WC}^{(20)}$. PartezaniRodrigues et al. ${ }^{(21)}$ reported that in the older adults the risk of frailty was significantly associated with WHtR but not with WC. However, no study investigated the mediating effect of WHtR on the association between BMI and the risk of frailty. Therefore, the present study aimed to confirm the hypothesis

Abbreviations: K-FRAIL, Korean version of the Fatigue, Resistance, Ambulation, Illnesses, and Loss of weight; WC, waist circumference; WHtR, waist:height ratio.

* Corresponding author: Professor Yongsoon Park, fax +82-2220-1856, email yongsoon@hanyang.ac.kr

$\dagger$ These authors contributed equally to this work. 
that the risk of frailty was positively associated with obesity, but the association was mediated by WHtR in the older women and men.

\section{Methods}

\section{Participants}

The present study used the data from the Korean Frailty and Aging Cohort Study (KFACS), a multicentre, longitudinal study of community-dwelling older adults ${ }^{(22)}$. Between June 2016 and November 2017, the participants of the KFACS were recruited from ten participating centres across the urban and rural areas of Korea on the basis of age- and sex-specific strata. The following participants were included in the study: those aged 70-84 years, those living independently at home, those with no plans to move out in the next 2 years and those having no serious problems with communication. Among the 3014 participants who completed the initial assessment, 2997 participants with non-missing data on frailty assessments were included in the present study. The study was conducted according to the guidelines in the Declaration of Helsinki ${ }^{(23)}$ and was approved by the institutional review board of Kyung Hee University (KHUH-2015-12-103-044) and the institutional review board of Hanyang University (HYI-18-167-1). All participants signed a written informed consent form.

\section{Data collection}

Data were obtained from the $\operatorname{KFACS}^{(22)}$ and included age, sex, height, weight, WC, educational level (less than middle school or more than high school), job (currently working, previously working or non-working), smoking (current, former or never), alcohol drinking (once or more times in a month) and living alone. Polypharmacy was defined as the regular use of five or more prescription medications. Cognitive impairment was defined as a Korean Mini-Mental State Examination score of less than $24^{(24)}$. Co-morbidity was defined as the number of self-reported diseases diagnosed by a physician (hypertension, dyslipidaemia, CVD, peripheral arterial disease, cerebrovascular disease, osteoarthritis, osteoporosis, respiratory disease, lung disease, thyroid disease, dementia, kidney disease and cancer). Disabilities were defined as dependence in at least one item in the Korean activities of daily living ${ }^{(25)}$ and Korean instrumental activities of daily living ${ }^{(26)}$.

Weight was measured to the nearest $0 \cdot 1 \mathrm{~kg}$ using a portable digital scale, and height was measured to the nearest $0 \cdot 1 \mathrm{~cm}$ using a measuring tape. The BMI was calculated as weight divided by square of the height. According to the criteria of the World Health Organization Asia-Pacific Region ${ }^{(27)}$, BMI was categorised as $<18.5 \mathrm{~kg} / \mathrm{m}^{2}, 18.5-<23 \mathrm{~kg} / \mathrm{m}^{2}, 23-<25 \mathrm{~kg} / \mathrm{m}^{2}$, $25-<30 \mathrm{~kg} / \mathrm{m}^{2}$ and $\geq 30 \mathrm{~kg} / \mathrm{m}^{2}$. The WC was measured at the middle point between the rib margin and iliac crests in a horizontal plane using an inelastic measuring tape. The WHtR $(\mathrm{cm} / \mathrm{cm})$ was calculated as WC divided by height with the median value of 0.557 as the cut-off point in the study participants. Dual-energy $\mathrm{X}$-ray absorptiometry was used to obtain appendicular skeletal muscle mass (ASM) of the four limbs, and ASM index was calculated as the sum of muscle mass in the four limbs $(\mathrm{kg})$ divided by the squared height $\left(\mathrm{m}^{2}\right)$.

\section{Frailty indices}

Frailty was defined using the K-FRAIL index ${ }^{(28)}$, a modified version of the published FRAIL scale ${ }^{(29)}$. The FRAIL scale exhibited the strongest predictive validity for disability and mortality compared with the Cardiovascular Health Study (CHS) index ${ }^{(30)}$. Since the K-FRAIL index is easier to measure compared with CHS because of interview-based test ${ }^{(31)}$, the K-FRAIL index was measured in all participants in the study. The K-FRAIL index assigns 1 point to each of the following five components. Fatigue was assessed by asking the participants for how much time during the preceding 4 weeks they felt tired, with responses of 'all of the time' or 'most of the time' scored as 1 point. Resistance was defined as difficulty in walking up to ten stair steps alone without resting and without aid, and ambulation was assessed by asking whether they had any difficulty in walking $300 \mathrm{~m}$ alone and without aid. Illness was defined as having five or more conditions among the following eleven conditions: hypertension, diabetes mellitus, chronic obstructive pulmonary disease, angina, myocardial infarction, heart failure, asthma, arthritis, stroke, renal disease and cancer. Loss of weight was recorded as loss of at least $5 \%$ of the body weight within the preceding year. Participants with a total score of 3 or higher are classified as frail.

\section{Statistical analysis}

Independent two-sample $t$ tests for continuous variables and $\chi^{2}$ tests for categorical variables were used to compare the differences in the characteristics and risk factors for frailty between older women and men. Continuous variables were presented as means and standard deviations, and categorical variables were expressed as the number of participants (percentage distribution). Pearson's correlation analysis was performed to determine the bivariate association between the main variables. To assess whether the association between BMI and risk of frailty weakened or became non-significant after adjusting for WHtR, binary logistic regression analysis was performed after adjusting for confounding factors. ANCOVA with least significant difference post hoc test was used in comparing the marginal means of lean mass by adjusting the confounding variables.

Moderation, mediation and moderated mediation analyses were performed using the Hayes PROCESS macro (models 1, 4 and 14$)^{(32)}$. For these analyses, independent, dependent and mediator variables or categorical covariates were designated as numerical variables or dummy variables. Additionally, moderator variables were recoded using indicator coding, with men as the reference category ${ }^{(33)}$. This macro used a bootstrapping strategy to test the validity of the indirect effects and calculate the $95 \%$ bias-corrected CI from 5000 bootstrap samples ${ }^{(34)}$. These analyses were considered significant if the CI excluded zero. In the multivariable models, the covariates showing a significance level below $0 \cdot 2$ were included in the final model ${ }^{(35)}$. All statistical tests were two sided according to a 0.05 significance level and performed using SPSS (version 25.0; SPSS Inc.). 
Table 1. Characteristics of women and men* (Mean values and standard deviations; percentages)

\begin{tabular}{|c|c|c|c|c|c|c|c|}
\hline & \multicolumn{2}{|c|}{ Total ( $n$ 2862) } & \multicolumn{2}{|c|}{ Women $(n$ 1482) } & \multicolumn{2}{|c|}{ Men $(n$ 1380) } & \multirow[b]{2}{*}{$P \dagger$} \\
\hline & Mean & SD & Mean & SD & Mean & SD & \\
\hline Age (years) & 75.96 & 3.89 & $75 \cdot 66$ & 3.85 & $76 \cdot 29$ & 3.91 & $<0.001$ \\
\hline Height $(\mathrm{cm})$ & $157 \cdot 88$ & 8.54 & 151.50 & $5 \cdot 27$ & $164 \cdot 72$ & 5.55 & $<0.001$ \\
\hline Weight (kg) & 61.03 & $9 \cdot 33$ & $57 \cdot 26$ & 7.93 & 65.08 & 9.00 & $<0.001$ \\
\hline BMI $\left(\mathrm{kg} / \mathrm{m}^{2}\right)$ & 24.46 & 3.01 & 24.92 & 3.03 & 23.96 & 2.92 & $<0.001$ \\
\hline \multicolumn{8}{|l|}{ BMI (\%) } \\
\hline$<18.5 \mathrm{~kg} / \mathrm{m}^{2}$ & \multirow{2}{*}{\multicolumn{2}{|c|}{$\begin{array}{l}1.9 \\
1.9\end{array}$}} & \multicolumn{2}{|c|}{0.9} & \multicolumn{2}{|c|}{3.0} & $<0.001$ \\
\hline $18.5-<23 \mathrm{~kg} / \mathrm{m}^{2}$ & & & \multicolumn{2}{|c|}{$25 \cdot 2$} & \multicolumn{2}{|c|}{33.7} & \\
\hline $23-<25 \mathrm{~kg} / \mathrm{m}^{2}$ & \multicolumn{2}{|c|}{28.5} & \multicolumn{2}{|c|}{28.5} & \multicolumn{2}{|c|}{28.6} & \\
\hline $25-<30 \mathrm{~kg} / \mathrm{m}^{2}$ & \multicolumn{2}{|c|}{$36 \cdot 1$} & \multirow{2}{*}{\multicolumn{2}{|c|}{$\begin{array}{c}39.9 \\
5.5\end{array}$}} & \multirow{2}{*}{\multicolumn{2}{|c|}{$\begin{array}{c}32.1 \\
2.6\end{array}$}} & \\
\hline$\geq 30 \mathrm{~kg} / \mathrm{m}^{2}$ & \multicolumn{2}{|c|}{$4 \cdot 1$} & & & & & \\
\hline $\mathrm{WC}^{-}(\mathrm{cm})$ & 87.83 & 8.49 & 87.05 & 8.37 & 88.66 & 8.56 & $<0.001$ \\
\hline $\mathrm{WHtR}(\mathrm{cm} / \mathrm{cm})$ & 0.56 & 0.06 & 0.58 & 0.06 & 0.54 & 0.05 & $<0.001$ \\
\hline Education (\%) & & & & & & & $<0.001$ \\
\hline$<$ High school & \multicolumn{2}{|c|}{$62 \cdot 3$} & \multicolumn{2}{|c|}{$76 \cdot 0$} & \multicolumn{2}{|c|}{$47 \cdot 7$} & \\
\hline$\geq$ High school & \multicolumn{2}{|c|}{37.7} & \multicolumn{2}{|c|}{$24 \cdot 0$} & \multicolumn{2}{|c|}{$52 \cdot 3$} & \\
\hline Currently working (\%) & \multicolumn{2}{|c|}{$26 \cdot 2$} & \multicolumn{2}{|c|}{$22 \cdot 1$} & \multicolumn{2}{|c|}{30.5} & $<0.001$ \\
\hline Smoking (\%) & & & & & & & $<0.001$ \\
\hline Current & \multicolumn{2}{|c|}{$5 \cdot 7$} & \multicolumn{2}{|c|}{0.9} & \multicolumn{2}{|c|}{10.9} & \\
\hline Former & 33 & & & & & & \\
\hline Never & 60 & & & & & & \\
\hline Alcohol drinking (\%) & 37 & & & & & & $<0.001$ \\
\hline Living alone (\%) & 22 & & & & & & $<0.001$ \\
\hline Polypharmacy (\%) & 33 & & & & & & 0.001 \\
\hline Cognitive impairment (\%) & 20 & & & & & & $<0.001$ \\
\hline Co-morbidity (\%) & & & & & & & $<0.001$ \\
\hline 0 & 16 & & & & & & \\
\hline 1 & 26 & & & & & & \\
\hline$\geq 2$ & 56 & & & & & & \\
\hline ADL disability (\%) & 11 & & & & & & $<0.001$ \\
\hline IADL disability (\%) & 37 & & & & & & $<0.001$ \\
\hline Frailty (\%) & 12 & & & & & & $<0.001$ \\
\hline
\end{tabular}

WC, waist circumference; WHtR, waist:height ratio; $A D L$, activities of daily living; IADL, instrumental activities of daily living ${ }^{*}$ Number of participants was discordant; education ( $n$ 2861), at work ( $n$ 2860), smoking $(n$ 2861), alcohol drinking $(n$ 2852), polypharmacy ( $n$ 2848) and IADL ( $n$ 2857).

$\dagger P$ values were analysed using the independent $t$ test for continuous variables and $\chi^{2}$ test for categorical variables.

\section{Results}

\section{Characteristics of older women and men}

The prevalence of frailty was $12.3 \%$ and was higher in women than in men (Table 1). Women were younger; and had lower educational level, proportion of currently working, smoking, alcohol drinking, polypharmacy and instrumental activities of daily living disability. However, women had higher BMI, WHtR, proportion of living alone, cognitive impairment, co-morbidity and activities of daily living disability compared with men.

\section{Association between frailty and body composition}

Multivariable-adjusted regression analysis revealed that the risk of frailty was higher in all older individuals and in older men with BMI $<18.5 \mathrm{~kg} / \mathrm{m}^{2}$ before and after adjusting for WHtR (Table 2 ). Furthermore, the risk of frailty was higher in all older individuals and in older women with BMI $\geq 30 \mathrm{~kg} / \mathrm{m}^{2}$ before adjusting for WHtR, but the risk disappeared after adjusting for WHtR. On the other hand, the risk of frailty was lower in men with BMI $25-<30 \mathrm{~kg} / \mathrm{m}^{2}$ before adjusting for WHtR but lower in all older individuals and older women with BMI $25-<30 \mathrm{~kg} / \mathrm{m}^{2}$ after adjusting for WHtR.
When participants were divided into low and high WHtR groups according to the median value of 0.557 as cut-off point, the risk of frailty was higher in all older individuals and in older men with BMI $<18.5 \mathrm{~kg} / \mathrm{m}^{2}$ and low WHtR compared with those with BMI $18 \cdot 5-<23 \mathrm{~kg} / \mathrm{m}^{2}$ and low WHtR (Table 3). Moreover, the risk of frailty was higher in all older individuals and women with BMI $\geq 30 \mathrm{~kg} / \mathrm{m}^{2}$ and high WHtR than those with BMI $18 \cdot 5-<23 \mathrm{~kg} / \mathrm{m}^{2}$ and low WHtR. On the other hand, the risk of frailty was lower in men with BMI $25-<30 \mathrm{~kg} / \mathrm{m}^{2}$ and high WHtR compared with those with BMI $18 \cdot 5-<23 \mathrm{~kg} / \mathrm{m}^{2}$ and low WHtR.

\section{Mediating effects of waist:height ratio on the association between $B M /$ and frailty}

Bivariate correlations revealed that BMI was positively correlated with WHtR $(r 0.82, P<0.001)$ and frailty $(r$ 0.04, $P=0.024)$, and WHtR was positively correlated with frailty ( $r 0 \cdot 18, P<0 \cdot 001$ ) (online Supplementary Table S1). In the mediation analysis, the negative direct effect and the positive indirect effect of BMI on the score of frailty were significant in all older individuals, in older women and in older men (Fig. 1). On the other hand, the total effect of BMI on the score of frailty was positive in women but negative in men. Additionally, 
Table 2. Association between BMl and risk of frailty before and after adjusting for waist:height ratio (WHtR)* (Odds ratios and $95 \%$ confidence intervals)

\begin{tabular}{|c|c|c|c|c|c|c|c|c|c|c|c|c|}
\hline \multirow[b]{3}{*}{ BMI $\left(\mathrm{kg} / \mathrm{m}^{2}\right)$} & \multicolumn{6}{|c|}{ Model 1} & \multicolumn{6}{|c|}{ Model 2} \\
\hline & \multicolumn{2}{|c|}{ Total } & \multicolumn{2}{|c|}{ Women } & \multicolumn{2}{|r|}{ Men } & \multicolumn{2}{|c|}{ Total } & \multicolumn{2}{|c|}{ Women } & \multicolumn{2}{|c|}{ Men† } \\
\hline & OR & $95 \% \mathrm{Cl}$ & OR & $95 \% \mathrm{Cl}$ & OR & $95 \% \mathrm{Cl}$ & OR & $95 \% \mathrm{Cl}$ & OR & $95 \% \mathrm{Cl}$ & OR & $95 \% \mathrm{Cl}$ \\
\hline$<18.5$ & $3 \cdot 19$ & $1.55,6.57$ & 1.81 & $0.48,6.79$ & $4 \cdot 19$ & $1 \cdot 73,10 \cdot 17$ & 4.02 & $1.88,8.57$ & $2 \cdot 33$ & $0.60,9.05$ & 3.74 & $1 \cdot 36,10 \cdot 26$ \\
\hline $18 \cdot 5-<23$ & 1.0 & & 1.0 & & 1.0 & & 1.0 & & 1.0 & & 1.0 & \\
\hline $23-<25$ & 1.02 & $0.73,1.41$ & 0.92 & $0.61,1.40$ & $1 \cdot 10$ & $0.64,1.88$ & 0.86 & $0.60,1 \cdot 24$ & 0.72 & $0.45,1.14$ & $1 \cdot 19$ & $0.63,2.22$ \\
\hline $25-<30$ & 0.76 & $0.55,1.06$ & 0.92 & $0.62,1.36$ & 0.40 & $0.21,0.75$ & 0.56 & $0.36,0.87$ & 0.58 & $0.34,0.99$ & 0.45 & $0.19,1.07$ \\
\hline$\geq 30$ & 1.88 & $1 \cdot 11,3 \cdot 17$ & 1.86 & $1.01,3.42$ & 1.89 & $0.64,5.57$ & 1.08 & $0.51,2.28$ & 0.82 & $0.34,1.96$ & $2 \cdot 47$ & $0.51,11.97$ \\
\hline
\end{tabular}

$A D L$, activities of daily living; IADL, instrumental activities of daily living.

${ }^{*}$ For logistic regression model 1, it was adjusted for age, sex, education, alcohol drinking, polypharmacy, cognitive impairment, co-morbidity, ADL and IADL in total older individuals ( $n$ 2830); age, education, polypharmacy, cognitive impairment, co-morbidity, ADL and IADL in women ( $n$ 1465); age, education, alcohol drinking, living alone, polypharmacy, co-morbidity and IADL in men ( $n$ 1365). For logistic regression model 2, model 1 adjusted additionally for WHtR in total older adults, women and men.

† As WHtR was a NS covariate variable $(P=0.643)$, the Hosmer-Lemeshow test was significant $\left(\chi^{2}=16.689\right.$, df $\left.=8, P=0.034\right)$ for men after adjusting for WHtR in model 2 .

Table 3. Subgroup analysis of waist:height ratio (WHtR) on the association between BMl and risk of frailty ${ }^{*} \dagger$ (Odds ratios and $95 \%$ confidence intervals)

\begin{tabular}{|c|c|c|c|c|c|c|c|c|c|c|}
\hline & \multicolumn{10}{|c|}{ BMI $\left(\mathrm{kg} / \mathrm{m}^{2}\right)$} \\
\hline & \multicolumn{2}{|c|}{$<18.5$} & \multicolumn{2}{|c|}{$18 \cdot 5-<23$} & \multicolumn{2}{|c|}{$23-<25$} & \multicolumn{2}{|c|}{$25-<30$} & \multicolumn{2}{|c|}{$\geq 30$} \\
\hline & OR & $95 \% \mathrm{Cl}$ & OR & $95 \% \mathrm{Cl}$ & OR & $95 \% \mathrm{Cl}$ & OR & $95 \% \mathrm{Cl}$ & OR & $95 \% \mathrm{Cl}$ \\
\hline \multicolumn{11}{|l|}{ Total $(n$ 2830) } \\
\hline Low WHtR & 3.32 & $1.61,6.86$ & 1.0 & & 1.08 & $0.70,1.67$ & 0.29 & $0.10,0.82$ & $\mathrm{~N} / \mathrm{A}$ & \\
\hline High WHtR & $\mathrm{N} / \mathrm{A}$ & & 1.41 & $0.74,2.67$ & 1.07 & $0.71,1.61$ & 0.88 & $0.62,1.24$ & 2.00 & $1.17,3.42$ \\
\hline \multicolumn{11}{|c|}{ Women $(n$ 1465) } \\
\hline Low WHtR & 2.08 & $0.55,7.87$ & 1.0 & & 0.76 & $0.40,1.46$ & 0.70 & $0.23,2 \cdot 13$ & $\mathrm{~N} / \mathrm{A}$ & \\
\hline High WHtR & $\mathrm{N} / \mathrm{A}$ & & 1.74 & $0.86,3.50$ & $1 \cdot 21$ & $0.74,1.98$ & 1.08 & $0.70,1.67$ & $2 \cdot 13$ & $1 \cdot 13,4.01$ \\
\hline \multicolumn{11}{|l|}{ Men $(n$ 1365) } \\
\hline Low WHtR & $4 \cdot 16$ & $1 \cdot 70,10 \cdot 18$ & 1.0 & & 1.26 & $0.70,2 \cdot 27$ & $N / A$ & & $\mathrm{~N} / \mathrm{A}$ & \\
\hline High WHtR & $\mathrm{N} / \mathrm{A}$ & & $\mathrm{N} / \mathrm{A}$ & & 0.72 & $0.32,1.64$ & 0.50 & $0.26,0.95$ & 1.84 & $0.62,5.43$ \\
\hline
\end{tabular}

N/A, not applicable; ADL, activities of daily living; IADL, instrumental activities of daily living.

${ }^{*} \mathrm{~N} / \mathrm{A}$ was used if there were no frail or older adult participants.

† Confounding factors were age, sex, education, alcohol drinking, polypharmacy, cognitive impairment, co-morbidity, ADL and IADL in total older adults; age, education, polypharmacy, cognitive impairment, co-morbidity, ADL and IADL in women; age, education, alcohol drinking, living alone, polypharmacy, co-morbidity, ADL and IADL in men.

the mediating effect of WHtR on the score of frailty was positive in both women and men, but the score of frailty was associated with BMI positively in women but negatively in men, suggesting that the association was affected by sex (online Supplementary Fig. S1).

\section{Discussion}

The present study showed that the risk of frailty was higher in the older individuals with BMI $\geq 30 \mathrm{~kg} / \mathrm{m}^{2}$ than those with BMI $18-<23 \mathrm{~kg} / \mathrm{m}^{2}$ before adjusting for WHtR but not after adjusting for WHtR in total older individuals and women but not in men. The association between BMI and the risk of frailty was mediated by WHtR in the older individuals, particularly in women.

Consistent with the present study, the majority of previous studies reported that obesity (BMI $\geq 30 \mathrm{~kg} / \mathrm{m}^{2}$ ) was associated with a higher prevalence or incidence of frailty in the older adults without adjusting for abdominal obesity ${ }^{(8,10-16)}$. However, a few studies showed that the risk of frailty was not significantly higher in obese older adults ${ }^{(7,9,16)}$. This inconsistency could be partly because of the proportion of frailty among the female study participants. Older women tended to be more prone to obesity ${ }^{(36)}$ and had a higher prevalence of frailty compared with older men ${ }^{(37)}$. In previous studies reporting the absence of an association between obesity and frailty, the proportion of frailty in women (8-9 \%) was lower than that in the present study $(17 \%)^{(7,9)}$. Oestrogen levels declined after menopause, leading to a decrease in bone density, muscle mass and muscle strength but an increase in visceral adiposity ${ }^{(38)}$. Consistent with the findings of the present study, the prevalence of obesity was higher in women than in men ${ }^{(39)}$. Additionally, previous studies did not include abdominal obesity as a confounding factor to confirm the association between frailty and obesity ${ }^{(7-15)}$. In the present study, obesity was also associated with a higher risk of frailty before adjusting for $\mathrm{WHtR}$, but it was not associated with the risk of frailty after adjusting for WHtR. Previous epidemiological studies consistently reported that abdominal obesity defined by $\mathrm{WC}^{(8,9,16,17,40)}$ and $\mathrm{WHtR}^{(21)}$ was associated with a higher prevalence and incidence of frailty in the older adults. Older adults with high WC had high levels of oxidative stress markers, independent of BMI, suggesting a possible involvement of oxidative stress in the genesis of frailty ${ }^{(18,19)}$. Garcia-Esquinas et $a l .{ }^{(16)}$ reported that the association between obesity and the risk of frailty was not significant in the older adults after adjusting 

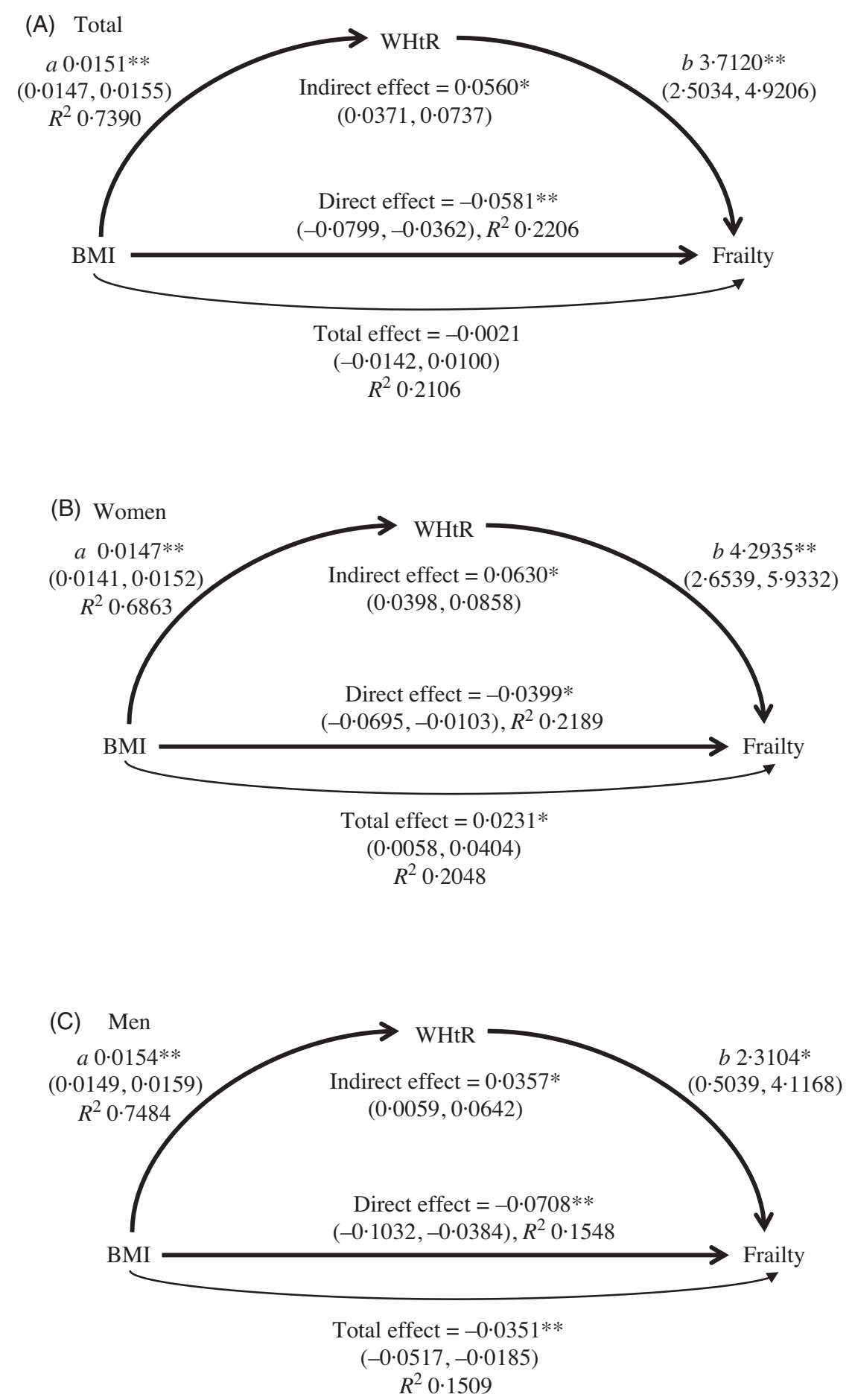

Fig. 1. Mediating effects of waist:height ratio $(\mathrm{WHtR})$ on the association between $\mathrm{BMl}$ and frailty score in total older adults $(\mathrm{A})$, women $(\mathrm{B})$ and men $(\mathrm{C})$. Unstandardised coefficients and $95 \%$ confidence intervals are presented: ' $a$ ' is the linear regression coefficient of the BMI-WHtR association and ' $b$ ' is that of the WHtR-frailty association. Adjusted confounding factors were age, sex, education, alcohol drinking, polypharmacy, cognitive impairment, activities of daily living (ADL) and instrumental activities of daily living (IADL) in total older adults; age, education, polypharmacy, cognitive impairment, ADL and IADL in women; age, education, alcohol drinking, living alone, polypharmacy, ADL and IADL in men. ${ }^{*} P<0.05,{ }^{* \star} P<0.001$.

for WC, suggesting that the association could be mediated by abdominal obesity. Moreover, Ferriolli et al. ${ }^{(9)}$ suggested that the WC may be one of the main factors that associate obesity with frailty in the older adults. However, Rodrigues et al. ${ }^{(21)}$ reported that the risk of frailty was positively associated with $\mathrm{WHtR} \geq 0.50$ but not with WC, suggesting that WHtR could be a better marker of abdominal obesity. Hirani et al. ${ }^{(41)}$ also reported that the risk of frailty was significantly higher in the older adults with sarcopenic obesity than in those with non-sarcopenic normal weight, but it was not significantly different from those with 
non-sarcopenic obesity. It is well known that sarcopenia, defined as an abnormal loss of muscle mass and strength, is one of the major causes for an increase in the risk of frailty ${ }^{(42)}$. Falsarella et al. ${ }^{(43)}$ reported that frail older individuals had a lower muscle mass and higher percentage of body fat compared with non-frail older individuals. In the present study, the obese older individuals had a higher lean mass compared with the older individuals with normal weight (online Supplementary Table S2).

In the present study, obesity was not associated with risk of frailty in men before and after adjusting for WHtR. The mediating effect of WHtR on the association between BMI and risk of frailty was shown only in women but not in men, suggesting that the mediating effect was moderated by sex (online Supplementary Fig. S1). In the present study, it could be impossible to detect the association between BMI and the risk of frailty in men, because there were only five frail men with obesity. Furthermore, according to the previous studies, men had a higher muscle mass compared with women ${ }^{(44)}$, and high muscle mass has been shown to be associated with lower risk of frailty in the older adults ${ }^{(45)}$. The present study also showed that men had more muscle mass compared with women (online Supplementary Table S2). Bigaard et al. ${ }^{(46)}$ reported that WC reflected visceral fat, whereas BMI reflected fat-free mass or fat deposit elsewhere, suggesting that BMI in men could not be associated with the risk of frailty because of the body composition effect. In the present study, the effect of BMI on frailty score was positive in women, but negative in men, suggesting that high muscle mass in men could attenuate the adverse effect of obesity through WHtR on the risk of frailty (online Supplementary Fig. S1).

The previous studies consistently suggested that underweight was associated with higher prevalence and incidence of frailty ${ }^{(6-10,12-15)}$. Underweight could have resulted from malnutrition, cachexia and sarcopenia, which were also associated with increased risk of frailty and represent low reserve capacity and weight loss, a key component of frailty ${ }^{(47)}$. In the present study, underweight was associated with higher risk of frailty in the older men before and after adjusting for WHtR, suggesting that WHtR was not associated with the risk of frailty in men who are underweight. However, in the present study, the risk of frailty was not higher in underweight women because of a small proportion of underweight women with frailty $(n$ 4).

Previous studies reported that overweight defined by BMI $25-29.9 \mathrm{~kg} / \mathrm{m}^{2}$ was associated with lower prevalence and incidence of frailty in the older adults ${ }^{(8-10,17)}$. Overweight was also associated with lower risk of mortality in the older adults, suggesting that overweight could represent a higher reserve capacity than those who are underweight or even normal weight ${ }^{(7)}$. Oreopoulos et $a l .{ }^{(48)}$ reported that a higher BMI in advanced age can actually be considered a protective factor against oxidative stress, inflammation, malnutrition, fractures and cognitive decline. Additionally, the negative association between BMI and mortality suggested a paradoxical association of overweight or obesity with reduced CVD-related mortality in the older adults ${ }^{(49)}$. In the present study, BMI $25-29 \cdot 9 \mathrm{~kg} / \mathrm{m}^{2}$ was associated with lower risk of frailty in all older individuals and in women after adjusting for WHtR.

The present study has a few limitations. First, the present study was composed of only a small proportion of frail older individuals. Second, the cross-sectional study design was not able to identify the causal association between BMI and WHtR with the risk of frailty. Third, although adjustments for confounders were made, unmeasured factors might affect the results of the present study.

In conclusion, the present study showed that the risk of frailty was higher in obese individuals, which was mediated by WHtR in older individuals, particularly older women, suggesting that abdominal obesity could increase the risk of frailty in women but not in men. A large population-based prospective observational study is needed to confirm whether the reduction in WHtR in obese older adults reduces the risk of frailty.

\section{Acknowledgements}

This work was supported by the National Research Foundation of Korea (NRF) grant funded by the Korean Government (MSIT) (NRF-2018R1A2B6002486) and a grant of the Korea Health Technology R\&D Project through the Korean Health Industry Development Institute (KHIDI) funded by the Ministry of Health \& Welfare, the Republic of Korea (grant number: HI15C3153).

The authors' contributions are as follows: M. K. performed the statistical analyses and prepared the first draft, Y. L. interpreted the results and edited the manuscript, E. K. assisted with statistical analyses and interpretation of results and edited the manuscript and Y. P. designed the study and finalised the manuscript. All authors read and approved the final manuscript.

The authors declare that there are no conflicts of interest.

\section{Supplementary material}

For supplementary material/s referred to in this article, please visit https://doi.org/10.1017/S0007114519002058

\section{References}

1. Fried LP, Ferrucci L, Darer J, et al. (2004) Untangling the concepts of disability, frailty, and comorbidity: implications for improved targeting and care. J Gerontol A Biol Sci Med Sci 59, M255-M263.

2. He W, Goodkind D \& Kowal P (2016) An aging world: 2015. International population reports. Washington DC: U.S. Census Bureau.

3. Kim KJ, Shin J, Choi J, et al. (2018) Discrepancies in the prevalence of known frailty scales: Korean Frailty and Aging Cohort Study. Ann Geriatr Med Res 22, 137-144.

4. Morley JE (2007) Weight loss in older persons: new therapeutic approaches. Curr Pharm Des 13, 3637-3647. 
5. Reinders I, Visser M \& Schaap L (2017) Body weight and body composition in old age and their relationship with frailty. Curr Opin Clin Nutr Metab Care 20, 11-15.

6. Sanchez-Garcia S, Sanchez-Arenas R, Garcia-Pena C, et al. (2014) Frailty among community-dwelling elderly Mexican people: prevalence and association with sociodemographic characteristics, health state and the use of health services. Geriatr Gerontol Int 14, 395-402.

7. Lee Y, Kim J, Han ES, et al. (2014) Frailty and body mass index as predictors of 3 -year mortality in older adults living in the community. Gerontology 60, 475-482.

8. Hubbard RE, Lang IA, Llewellyn DJ, et al. (2010) Frailty, body mass index, and abdominal obesity in older people.J Gerontol A Biol Sci Med Sci 65, 377-381.

9. Ferriolli E, Pessanha F, Moreira VG, et al. (2017) Body composition and frailty profiles in Brazilian older people: Frailty in Brazilian Older People Study-FIBRA-BR. Arch Gerontol Geriatr 71, 99-104

10. Rietman ML, Van Oostrom S, Picavet H, et al. (2018) The association between BMI and different frailty domains: a U-shaped curve? J Nutr Health Aging 22, 8-15.

11. Sheehan KJ, O'Connell MD, Cunningham C, et al. (2013) The relationship between increased body mass index and frailty on falls in community dwelling older adults. $B M C$ Geriatr 13, 132.

12. Lopez SR, Montero P \& Carmenate M (2014) Educational inequalities and frailty in Spain: what is the role of obesity? J Frailty Aging 3, 120-125.

13. Woods NF, LaCroix AZ, Gray SL, et al. (2005) Frailty: emergence and consequences in women aged 65 and older in the Women's Health Initiative Observational Study. J Am Geriatr Soc 53, 1321-1330.

14. Blaum CS, Xue QL, Tian J, et al. (2009) Is hyperglycemia associated with frailty status in older women? J Am Geriatr Soc 57, 840-847.

15. Blaum CS, Xue QL, Michelon E, et al. (2005) The association between obesity and the frailty syndrome in older women: the Women's Health and Aging Studies. J Am Geriatr Soc 53 , 927-934.

16. Garcia-Esquinas E, Jose Garcia-Garcia F, Leon-Munoz LM, et al. (2015) Obesity, fat distribution, and risk of frailty in two population-based cohorts of older adults in Spain. Obesity (Silver Spring) 23, 847-855.

17. Liao Q, Zheng Z, Xiu S, et al. (2018) Waist circumference is a better predictor of risk for frailty than BMI in the communitydwelling elderly in Beijing. Aging Clin Exp Res 30, 1319-1325.

18. Weinbrenner T, Schröder H, Escurriol V, et al. (2006) Circulating oxidized LDL is associated with increased waist circumference independent of body mass index in men and women. Am J Clin Nutr 83, 30-35.

19. Serviddio G, Romano A, Greco A, et al. (2009) Frailty syndrome is associated with altered circulating redox balance and increased markers of oxidative stress. Int J Immunopathol Pharmacol 22, 819-827.

20. Swainson MG, Batterham AM, Tsakirides C, et al. (2017) Prediction of whole-body fat percentage and visceral adipose tissue mass from five anthropometric variables. PLOS ONE 12, $\mathrm{e} 0177175$.

21. Rodrigues RP, Fhon JS, Huayta VR, et al. (2017) Frailty syndrome and anthropometric measurements in the elderly living at home. J Aging Res Clin Pract 6, 133-138.

22. Won CW, Lee Y, Choi J, et al. (2016) Starting construction of frailty cohort for elderly and intervention study. Ann Geriatr Med Res 20, 114-117.

23. Dale O \& Salo M (1996) The Helsinki Declaration, research guidelines and regulations: present and future editorial aspects. Acta Anaesthesiol Scand 40, 771-772.
24. Kang Y, Na DL \& Hahn S (1997) A validity study on the Korean Mini-Mental State Examination (K-MMSE) in dementia patients. J Korean Neurol Assoc 15, 300-308.

25. Won CW, Rho YG, Kim SY, et al. (2002) The validity and reliability of Korean Activities of Daily Living (K-ADL) scale. J Korean Geriatr Soc 6, 98-106.

26. Won CW, Rho YG, Duk S, et al. (2002) The validity and reliability of Korean Instrumental Activities of Daily Living (K-IADL) scale. J Korean Geriatr Soc 6, 273-280.

27. World Health Organization (2000) The Asia-Pacific Perspective: Redefining Obesity and Its Treatment. Sydney: Health Communications Australia.

28. Jung HW, Yoo HJ, Park SY, et al. (2016) The Korean version of the FRAIL scale: clinical feasibility and validity of assessing the frailty status of Korean elderly. Korean JIntern Med 31, 594-600.

29. Morley JE, Malmstrom T \& Miller D (2012) A simple frailty questionnaire (FRAIL) predicts outcomes in middle aged African Americans. J Nutr Health Aging 16, 601-608.

30. Malmstrom TK, Miller DK \& Morley JE (2014) A comparison of four frailty models. J Am Geriatr Soc 62, 721-726.

31. Woo J, Leung J \& Morley JE (2012) Comparison of frailty indicators based on clinical phenotype and the multiple deficit approach in predicting mortality and physical limitation. $J \mathrm{Am}$ Geriatr Soc 60, 1478-1486.

32. Hayes AF (2013) Introduction to Mediation, Moderation, and Conditional Process Analysis: A Regression-based Approach. New York: The Guilford Press.

33. Hayes AF \& Preacher KJ (2014) Statistical mediation analysis with a multicategorical independent variable. Br J Math Stat Psychol 67, 451-470.

34. Hayes AF (2009) Beyond Baron and Kenny: statistical mediation analysis in the new millennium. Commun Monogr 76, 408-420.

35. Greenland S \& Pearce N (2015) Statistical foundations for model-based adjustments. Annu Rev Public Health 36, 89-108.

36. Ogden CL, Yanovski SZ, Carroll MD, et al. (2007) The epidemiology of obesity. Gastroenterology 132, 2087-2102.

37. Collard R, Boter H, Schoevers R, et al. (2012) Prevalence of frailty in community-dwelling older persons: a systematic review. J Am Geriatr Soc 60, 1487-1492.

38. Messier V, Rabasa-Lhoret R, Barbat-Artigas S, et al. (2011) Menopause and sarcopenia: a potential role for sex hormones. Maturitas 68, 331-336.

39. Hong KS, Bang OY, Kang DW, et al. (2013) Stroke statistics in Korea: part I. Epidemiology and risk factors: a report from the Korean stroke society and clinical research center for stroke. J Stroke 15, 2-20.

40. Liu LK, Lee WJ, Chen LY, et al. (2015) Association between frailty, osteoporosis, falls and hip fractures among communitydwelling people aged 50 years and older in Taiwan: results from I-Lan Longitudinal Aging Study. PLOS ONE 10, e0136968.

41. Hirani V, Naganathan V, Blyth F, et al. (2017) Longitudinal associations between body composition, sarcopenic obesity and outcomes of frailty, disability, institutionalisation and mortality in community-dwelling older men: The Concord Health and Ageing in Men Project. Age Ageing 46, 413-420.

42. Yu Z, Ruan Q, \& D'Onofrio G (2017) From Sarcopenia to Frailty: the pathophysiological basis and potential target molecules of intervention. In Frailty and Sarcopenia-Onset, Development and Clinical Challenges, pp. 56-57 [Y Dionyssiotis, editor]. Croatia: InTech.

43. Falsarella GR, Gasparotto LPR, Barcelos CC, et al. (2015) Body composition as a frailty marker for the elderly community. Clin Interv Aging 10, 1661-1666.

44. Bredella MA (2017) Sex differences in body composition. In Sex and Gender Factors Affecting Metabolic Homeostasis, Diabetes and Obesity, p. 12 [FM Jarvis, editor]. Basel, Switzerland: Springer. 
45. Meng L, Shi H, Shi J, et al. (2019) Differences in clinical characteristics, muscle mass, and physical performance among different frailty levels in Chinese older men. Chin Med J $\mathbf{1 3 2}$ 352-355.

46. Bigaard J, Tjønneland A, Thomsen BL, et al. (2003) Waist circumference, BMI, smoking, and mortality in middle-aged men and women. Obes Res 11, 895-903.
47. Rolland Y, Van Kan GA, Gillette GuyonnetS, et al. (2011) Cachexia versus sarcopenia. Curr Opin Clin Nutr Metab Care 14, 15-21.

48. Oreopoulos A, Kalantar-Zadeh K, Sharma AM, et al. (2009) The obesity paradox in the elderly: potential mechanisms and clinical implications. Clin Geriatr Med 25, 643-659.

49. Kim TN (2018) Elderly obesity: is it harmful or beneficial? JObes 27, 84-92. 